\title{
Peertechz
}

\section{The Role of Speech Therapists in Olfactory Rehabilitation: a Short Report on Ten Cases of Total Laryngectomy}

Received: 11 November, 2020

Accepted: 07 December, 2020

Published: 09 December, 2020

*Corresponding author: Vittorio Grill, Department of Life Sciences, University of Trieste, Via Manzoni 16, I-34138 Trieste, Italy, Tel: +39040 5586006; Fax: +39 040 5586016; E-mail: grill@units.it

Keywords: Total laryngectomy; Olfactory

rehabilitation; Speech therapist

https://www.peertechz.com

Check for updates

\section{Michelle Passerini', Martina Da Re' ${ }^{1}$, Sonia Antoni' , Cristina Pierluca ${ }^{1}$, Giancarlo Tirelli ${ }^{1,2}$ and Vittorio Grill ${ }^{1,3 *}$ \\ ${ }^{1}$ School of Speech Therapy Trieste-Udine, Department of Medical, Surgical and Health Sciences, University of Trieste, Italy \\ ${ }^{2}$ Operative Unit of Otolaryngology, Department of Medical, Surgical and Health Sciences, University of Trieste, Italy}

${ }^{3}$ Chair of Human Anatomy, Department of Life Sciences, University of Trieste, Italy

\begin{abstract}
Introduction: Total Laryngectomy (TL) has to be performed in advanced hypopharyngeal or laryngeal cancer, with the consequence of both the loss of the vocal functions and the impairment or loss of smell and taste. Since less attention is turned towards the recovery of the olfactory function compared to the vocal rehabilitation, the Nasal Airflow-Induced Maneuver (NAIM) was proposed as a useful smell recovery method. In this short note, we report the results of the olfactory rehabilitation procedures by performing the NAIM technique in ten total laryngectomized patients with the evaluation of both the subjective olfactory detection and the olfactory discrimination, recognition and identification of odorous substances and we would emphasize the role of speech therapists in this rehabilitative procedure.
\end{abstract}

Materials and methods: Ten total laryngectomized patients were proposed to undergo an olfactory rehabilitation cycle performed by speech therapists by employing the NAIM technique. The subjective olfactory detection was evaluate at three different time intervals during the rehabilitation cycle. The olfactory discrimination, recognition and identification capability was considered both during and 12 moths after the end of the rehabilitation treatment.

Results: The NAIM performed together with the speech therapist ameliorated significantly all olfactory functions. evidence and the functional recovery was confirmed 12 months after the end of the rehabilitation cycle, thus the role of speech therapists appears relevant in both the olfactory recovery and the maintenance of the regained olfactory capability.

Conclusion: The role of speech therapists should be aimed not exclusively towards the recovery of the vocal functions, but also towards the recovery of the olfactory functions. Speech therapists are able to adjust the original NAIM method to the specific need of each patient. Therefore, the role of speech therapists should be considered highly relevant to regain the olfactory function to allow again a good quality of life even after TL surgery.

\section{Abbreviations}

TL: Total Laryngectomy; NAIM: Nasal Airflow-Inducing Maneuver

\section{Introduction}

Speech therapists work to prevent, assess and treat speech, language, cognitive-communication and swallowing disorders in children and adults in order to solve the consequence of various pathological conditions. Among these ones, advanced laryngeal and hypopharyngeal cancer requests the surgical procedure of Total Laryngectomy (TL), consisting on the removal of the whole larynx with the entire hyoid bone. At present in most clinical structures the speech therapist intervention after TL is exclusively aimed to recovery the specific speech activity 
by performing various alaryngeal speech techniques, i.e. by means of the oesophageal voice as well as by the application of voice prostheses. However, in TL the whole disconnection of the upper and lower airways causes deterioration of many other physiological functions in addition to the loss of the vocal one, including a remarkable impairment, and even total loss, of the specific senses of smell and taste [1-3], that are strictly mutually associated by various anatomical and functional features. Unfortunately less attention is generally turned to this remarkable consequence of $\mathrm{TL}$, that causes an evident decrease in the quality of life in total laryngectomized patients. As far as the recovery of the olfactory function is concerned, the NAIM technique (the so named "Polite Yawning") [3] is widely suggested for this aim and also adequate modifications of the original procedure were proposed to obtain better and more durable results in the regain of an acceptable olfactory function [4]. The NAIM consists of an extended yawning movement together with a simultaneous lowering of the jaw, as well as of both the body and the base of the tongue and the soft palate, while keeping the lips securely closed. In this short report, we would remark the peculiar role played by speech therapists in the rehabilitation of the olfactory function in ten cases of total laryngectomized patients by training them to the NAIM procedure and to stimulate them to frequently repeat the specific NAIM exercise both during the specific olfactory rehabilitation cycle and after the end of a 4-week treatment period.

\section{Materials and methods}

Ten total laryngectomized male patients, aged between 58 and 76 years (mean age $67.30 \pm 6.04$, median age 67 ), free from both nasal and pharyngeal concurrent pathologies, as well as from anatomical anomalies, agreed to the proposal to undergo an olfactory rehabilitation cycle performed by speech therapists in addition to the classic voice recovery treatment. All of them underwent total laryngectomy in different periods for advanced laryngeal cancer; none of them underwent either chemotherapy or radiotherapy after surgery and had at least a 2-year follow up period to evaluate stabilized late adverse effects. All patients already underwent voice rehabilitation and they were using different alaryngeal speech techniques, i.e. the oesophageal voice or voice prostheses. All patients were trained by speech therapists to the NAIM [3], to enable air and odorous substances to reach again the olfactory mucosa in the nasal cavities. The olfactory capability was evaluated with both the subjective olfactory perception and the olfactory discrimination, recognition and identification.

\section{Speech therapist intervention in the olfactory rehabilita- tion technique}

Sessions of the olfactory rehabilitation cycle (each with a duration of $45 \mathrm{~min}$ ) started at the end of clinical preliminar evaluations to exclude the presence of concurrent both nasal and pharyngeal pathologies, as well as anatomical anomalies, and were performed three times a week for four weeks (4-week treatment period), i.e. for a total of twelve 45 -min sessions. Speech therapists trained patients to the NAIM [3] to provoke a negative pressure both in the oral cavity and the oropharynx to restore the nasal airflow, thus to enable odorous substances to reach again the Schultz's olfactory neuronal receptors located within the olfactory mucosa. Patient were required to repeat the NAIM exercises several times at every rehabilitation unit to acquire a good expertise and they were also stimulated to actively use the NAIM as often as possible, as well as in particular after the end of the 4-week rehabilitation cycle.

\section{Subjective olfactory detection}

Tests of olfactory detection were performed at three time intervals, i.e. before the beginning of the olfactory rehabilitation cycle, after the third rehabilitation unit, i.e. at the end of the first rehabilitation week, and at the end of the olfactory rehabilitative intervention. A 4\% 1-butanol solution in di-propylene glycol was employed to assess the subjective olfactory detection capability of each patient. Patients were asked by speech therapists to express their subjective detected smell intensity by means of a numeric score between 0 (no olfactory detection) and 10 (maximum of olfactory detection). Score mean values of the voted subjective smell intensity at each considered period were calculated and statistical analyses were performed by considering the Student's t-test with significant differences verified when $\mathrm{p}<0.01$.

\section{Olfactory discrimination, recognition and identification}

Patients underwent olfactory discrimination, recognition and identification tests of different odorous substances at three different periods, i.e. before beginning the 4-week olfactory rehabilitation cycle, at the end of the fourth week of treatment cycle and finally 12 months after the end of the rehabilitation treatment. The olfactory discrimination, recognition and identification test consisted in sniffing sticks each containing a specific odorant. Each patient was presented with 26 sticks and everyone had to choose the correct answer among four choices in a questionary. Presentation of each stick was separated by at least 30 seconds. Mean values of the correctly identified substances were calculated. Statistical analyses were performed by considering the Student's t-test with significant differences verified when $\mathrm{p}<0.01$

\section{Results and discussion}

\section{Speech therapist intervention in the olfactory rehabilita- tion technique}

Three 45 min-training sessions were performed weekly by speech therapists and those were repeated during a 4-week period for a total of twelve 45-min sessions. At the beginning of the olfactory rehabilitation cycle speech therapists explained the anatomical and functional changes caused by TL and teached the laryngectomized patients the NAIM technique. Most patients learned the NAIM movements already during the first session of the rehabilitation cycle, however a minority of them found particular difficulties in NAIM application, therefore they were suggested to provide some minimal modifications to the original NAIM technique: in particular, for the patients speaking with the oesophageal voice, they were suggested to reproduce the same action they usually performed for the oesophageal speech. Every patient followed carefully every 
rehabilitation phase, thus every patient was able to acquire a correct NAIM technique either by applying the original method [3] or by using the above mentioned modified method with an adaptation suggested by speech therapists for each specific patient. We performed sessions with a longer duration (45 min vs. $30 \mathrm{~min}$ ) than that proposed in the original method [3] and we assessed the progress of patients in learning of the NAIM movements, as well as the recovery of the olfactory detection capability, by presenting our patients the 4\% 1-butanol solution in di-propylene glycol, i.e. with a subjective detection method, instead of the employ of a digital manometer evaluating a negative air pressure within the oropharyngeal space. During the olfactory rehabilitation cycle, we could observe a significant amelioration of the olfactory detection capability already after the third olfactory rehabilitation unit in comparison to the olfactory detection test performed before the beginning of the olfactory rehabilitation treatment. Moreover, we think particularly relevant the evidence that the olfactory rehabilitation benefits were preserved 12 months after the end of the rehabilitation treatment, showing that patients followed continuously the suggestions of speech therapists to perform the specific NAIM exercises with or without the modifications proposed for each laryngectomized patient.

\section{Subjective olfactory detection}

Data of this experimental phase are reported in Table 1. Before beginning the olfactory rehabilitation cycle, the mean value of the olfactory detection was $3.40 \pm 1.71$ in a score beteween a minimum of 1 and a maximum of 6 . After the third rehabilitation unit, the mean value of the olfactory detection was $5.70 \pm 1.25$, in a score between 4 and 7 . Finally, at the end of the rehabilitation cycle ( 4 weeks), the mean value of the olfactory detection increased up to $8.10 \pm 0.74$, in a score between 7 to 9 . By performing the paired Student's t-test, the significance of the differences between the mean values of the subjective olfactory detection without any rehabilitative intervention and those after the third olfactory rehabilitation session, as well as between this one and the olfactory sensibility at the end of the rehabilitative intervention, were evidenced for a level of $\mathrm{p}<0.01$ and it was considered as statistically significant. Therefore, it is evident that there was a significant amelioration of the subjective olfactory detection already after the third rehabilitative unit suggesting a peculiar role of the speech therapist intervention already in the earliest phase of the olfactory rehabilitation cycle, i.e. in the first week of the treatment. Moreover it appears particularly interesting, since it showed the very useful activity of speech therapists in teaching their laryngectomized patients the exercises of the NAIM procedure, even considering the progressive increase of the subjective olfactory detection capability during the 4-week rehabilitation cycle.

\section{Olfactory discrimination, recognition and identification}

Data of this experimental phase are reported in Table 2 and graphically presented in Figure 1. Patients underwent the first olfactory discrimination, recognition and identification test of odorous substances before performing the first olfactory rehabilitative intervention, but after the first subjective olfactory detection one. The first olfactory discrimination and recognition analysis revealed a mean value of $5.60 \pm 1.17$ of correctly identified substances among a total of 26 ones. At the end of the olfactory rehabilitation period ( 4 weeks), the mean value of correctly identified substances was $12.00 \pm 1.94$. A third olfactory discrimination, recognition and identification test were performed 12 months after the end of the smell rehabilitative procedure: the mean value of the correctly

Table 1: Subjective olfactory detection scores at three different time intervals during the 4-week olfactory rehabilitation cycle.

\begin{tabular}{|c|c|c|c|}
\hline Patients & $\begin{array}{c}\text { Subjective olfactory } \\
\text { detection scores } \\
\text { without smell } \\
\text { rehabilitation }\end{array}$ & $\begin{array}{c}\text { Subjective olfactory } \\
\text { detection scores after the } \\
\text { third rehabilitation session } \\
\text { (day 7) }\end{array}$ & $\begin{array}{c}\text { Subjective olfactory } \\
\text { detection scores at } \\
\text { the end of } \\
\text { 4-week rehabilitation } \\
\text { cycle }\end{array}$ \\
\hline 1 & 2 & 4 & 7 \\
\hline 2 & 3 & 5 & 8 \\
\hline 3 & 1 & 5 & 7 \\
\hline 4 & 4 & 6 & 8 \\
\hline 5 & 4 & 7 & 8 \\
\hline 6 & 5 & 7 & 8 \\
\hline 7 & 3 & 5 & 9 \\
\hline 8 & 1 & 4 & 8 \\
\hline 9 & 6 & 7 & $8,10 \pm 0.74$ \\
\hline 10 & 5 & $5,70 \pm 1.25$ & 9 \\
\hline Mean & $3.40 \pm 1.71$ & & 9 \\
\hline Values & & 7 & 9 \\
\hline
\end{tabular}

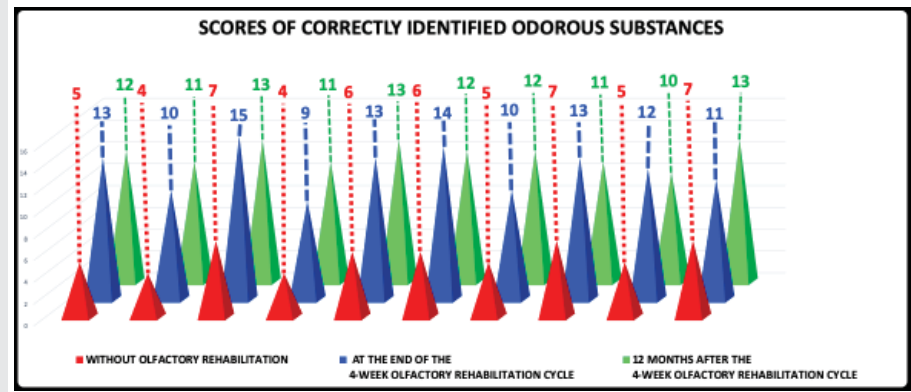

Figure 1: Scores of correctly identified odorous substances out of 26 sniffing sticks. Note the relevant increase of correctly identified substances at the end of the rehabilitation cycle in comparison to the scores observed at the beginning of the olfactory rehabilitation treatment. On the contrary no relevant differences was observed 12 months after the end of the rehabilitation period.

Table 2: Scores of correctly identified odorous substances out of 26 sniffing sticks at three different time intervals during and after the 4-week olfactory rehabilitation cycle.

\begin{tabular}{|c|c|c|c|}
\hline Patients & $\begin{array}{c}\text { Without olfactory } \\
\text { rehabilitation }\end{array}$ & $\begin{array}{c}\text { At the end of the } \\
\text { 4-week olfactory } \\
\text { rehabilitation cycle }\end{array}$ & $\begin{array}{c}\mathbf{1 2} \text { months after the } \\
\text { 4-week olfactory } \\
\text { rehabilitation cycle }\end{array}$ \\
\hline 1 & 5 & 13 & 12 \\
\hline 2 & 4 & 10 & 11 \\
\hline 3 & 7 & 15 & 13 \\
\hline 4 & 4 & 9 & 11 \\
\hline 5 & 6 & 13 & 13 \\
\hline 6 & 6 & 14 & 12 \\
\hline 8 & 5 & 10 & 12 \\
\hline 9 & 7 & 13 & 11 \\
\hline 10 & 5 & 12 & 10 \\
\hline Mean & 7 & 11 & 13 \\
\hline Values & $5,60 \pm 1,17$ & $12,00 \pm 1,94$ & $11,80 \pm 1,03$ \\
\hline
\end{tabular}


identified substances was $11.80 \pm 1.03$. By performing the paired Student's t-test, a significant difference $(\mathrm{p}<0.01)$ was observed between data at the end of the fourth rehabilitation week and those at the beginning of the 4-week olfactory rehabilitation cycle. On the contrary no significant difference was shown between data at the end of 4-week rehabilitation cycle and those observed 12 months after its conclusion: it appears very interesting, since it showed that the regain of a good olfactory function was also preserved 12 months after the end of the rehabilitation treatment and confirmed the relevance of the speech therapist intervention also in stimulating patients to continuously perform the specific NAIM exercises. Our data are also in accord to those reported by other investigators [4], even if they performed less training session (five 45-min sessions during a 5-week period). However, in our experience, we also observed that patients revealed some difficulties in the discrimination and recognition of some specific odorous substances perhaps because of a higher number of proposed sniffing sticks (26 vs. 16): although most substances were already well known by laryngectomees, however there were also other substances that were not well recognized by them, therefore we could assume that some patients could have reached better results in recognizing the proposed substances, if the classic odor detection test 16-stick test had been performed $[1,5]$.

\section{Conclusion}

Data reported in the present short communication showed that the rehabilitation of the olfactory functions should be highly considered in the follow up of patients undergone TL. The NAIM procedure was quickly learned by our ten laryngectomized patients and they continuously performed the specific NAIM exercises, since they were much motivated both themselves and by their speech therapists. In the follow up of
TL the speech therapist role should be aimed not exclusively towards the recovery of the vocal functions, but also towards the recovery of the olfactory detection, perception, discrimination and identification. We confirmed the great usefulness of the NAIM procedure and speech therapists were able to adjust the original method to the specific need of each patient. Therefore, the role of speech therapists should be considered highly relevant to regain the olfactory function to allow again a good quality of life even after TL surgery.

\section{Acknowledgement}

This work was partially supported by grants from the University of Trieste, RESRIC-Grill.

\section{References}

1. van Dam FSAM, Hilgers FJM, Emsbroek G, Touw Fl, van As CJ, et al. (1999) Deterioration of olfaction and gustation as consequence of total laryngectomy. Laryngoscope 109: 1150-1155. Link: https://bit.ly/3giaxn5

2. Riva G, Sensini M, Corvino A, Pecorari G, Garzaro M (2017) Smell and taste impairment after total laryngectomy. Ann Otol Rhinol Laryngol 126: 548-554. Link: https://bit.ly/3mS8993

3. Hilgers FJM, van Dam FSAM, Keyzers S, Koster MN, van As CJ, et al. (2000) Rehabilitation of olfaction after laryngectomy of a nasal airflow-inducing maneuver. The "Polite Yawning" technique. Arch Otolaryngol Head Neck Surg 126: 726-732. Link: https://bit.ly/39UQNot

4. Longobardi Y, Parrilla C, Di Cintio G, De Corso E, Marenda ME, et al. (2020) Olfactory perception rehabilitation after total laryngectomy (OPRAT): proposal of a new protocol based on training of sensory perception skills. Eur Arch Otorhinolaryngol 277: 2095-2105. Link: https://bit.ly/3IZl1cl

5. Hulshoff Pol HE, Hijman R, Baaré WF, van Ree JM (1998) Effects of context on judgement of odor intensities in humans. Chem Senses 23: 131-135. Link: https://bit.ly/2JWLCcu

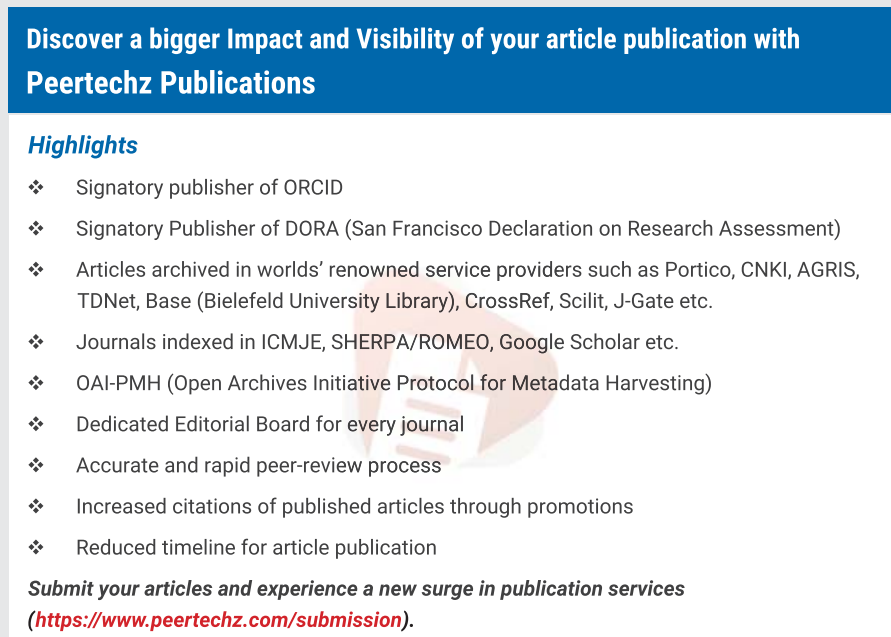

Peertechz journals wishes everlasting success in your every endeavours.

Copyright: (c) 2020 Passerini M, et al. This is an open-access article distributed under the terms of the Creative Commons Attribution License, which permits unrestricted use, distribution, and reproduction in any medium, provided the original author and source are credited. 